EUROPHYSICS LETTERS

10 June 1998

Europhys. Lett., 0 (0), pp. 0-0 (1998)

\title{
A Lattice Boltzmann Model of Ternary Fluid Mixtures
}

\author{
A. Lamura ${ }^{1}$, G. Gonnella ${ }^{1}$ and J. M. Yeomans ${ }^{2}$ \\ ${ }^{1}$ Dipartimento di Fisica, Universitá di Bari, Istituto Nazionale di Fisica della Materia, \\ Unità di Bari, and Istituto Nazionale di Fisica Nucleare, Sezione di Bari, \\ via Amendola 173, 70126 Bari, Italy \\ ${ }^{2}$ Department of Physics, Theoretical Physics, 1 Keble Rd., Oxford OX1 3NP, England.
}

(received ; accepted )

PACS. 47.20.Ma- Interfacial instability.

PACS. 05.70.Ln - Nonequilibrium thermodynamics, irreversible processes.

PACS. 83.10.Lk - Multiphase flows.

\begin{abstract}
A lattice Boltzmann model is introduced which simulates oil-water-surfactant mixtures. The model is based on a Ginzburg-Landau free energy with two scalar order parameters. Diffusive and hydrodynamic transport is included. Results are presented showing how the surfactant diffuses to the oil-water interfaces thus lowering the surface tension and leading to spontaneous emulsification. The rate of emulsification depends on the viscosity of the ternary fluid.
\end{abstract}

Introduction. - The addition of surfactant to a binary mixture of oil and water can produce many different complex structures on a mesoscopic length scale. The surfactant molecules move to the interface and lower the oil-water interfacial tension. This can result in, for example, lamellar, micellar, microemulsion or hexagonal arrangements of the oil and water domains 11. 2.

The equilibrium behaviour of such amphiphilic systems is well understood. However the dynamics of the self-assembly of the mesoscale phases and their rheology are less well investigated. This is a difficult problem because of the interplay between several relevant transport mechanisms, the diffusion of the constituent components and their hydrodynamic flow. To date models of amphiphilic rheology which treat hydrodynamic effects include time-dependent Ginzburg-Landau approaches 3, 4, molecular dynamics]5] and a lattice gas cellular automaton scheme based on microscopic interactions [6, 7].

The aim of this Letter is to introduce an alternative numerical scheme that can model the dynamics of amphiphilic systems in such a way that diffusive and hydrodynamic mechanisms are included. The numerical approach that we use is lattice Boltzmann simulations which have emerged as a useful tool to study the dynamics of complex fluids 8]. We base our approach on that described by Orlandini et. al. [9, 10] where the correct equilibrium of the fluid is imposed by choosing an appropriate free energy and including it in such a way that the fluid spontaneously reaches the equilibrium described by its minimum.

Previous lattice Boltzmann models of amphiphilic systems have been based on a single order

Typeset using EURO-TEX 
parameter, that of the phase separating binary fluid [11, 12]. The effect of the amphiphilic molecules has been mimiced by varying the surface tension in the input free energy. Although this approach proved successful it has the disadvantage of not including the surfactant dynamics explicitly.

We first give a description of the method and then present results showing how the surface tension of the binary fluid interface is lowered by surfactant at a rate which depends on the surfactant diffusion constant. As the surface tension becomes negative, this leads to the break-up of the interface and to spontaneous emulsification 13 to a lamellar phase.

The Lattice-Boltzmann Scheme. - We consider a Ginzburg-Landau model defined by the free energy functional [3, 14] which depends on two scalar order parameters $\phi(\mathbf{r})$ and $\rho(\mathbf{r})$

$$
\begin{aligned}
\mathcal{F}[\phi, \rho]= & \int d \mathbf{r}\left[\frac{a}{2} \phi^{2}+\frac{b}{4} \phi^{4}+\frac{\kappa}{2}(\nabla \phi)^{2}+\frac{c}{2}\left(\nabla^{2} \phi\right)^{2}+\frac{\alpha}{2} \rho^{2}+\frac{\lambda}{2}(\nabla \rho)^{2}+\frac{\gamma}{2}\left(\nabla^{2} \rho\right)^{2}\right. \\
& \left.+\beta_{1} \phi \rho^{2}+\beta_{2} \phi^{2}\left(\nabla^{2} \rho\right)+\beta_{3} \rho \phi\left(\nabla^{2} \phi\right)\right] .
\end{aligned}
$$

$\phi(\mathbf{r})$ and $\rho(\mathbf{r})$ can be identified, respectively, with the local density difference of oil and water and with the difference of local surfactant concentration from its average $\bar{\rho}$. $\bar{\rho}$ enters the model via the parameter $\kappa . \kappa$ is positive for small surfactant concentration. As $\kappa$ decreases and eventually becomes negative, $\bar{\rho}$ increases.

The thermodynamic variables that we will need are the chemical potential difference between oil and water $\Delta \mu$, the chemical potential $\Lambda$ of the surfactant and the pressure tensor $P_{\alpha \beta}$. The chemical potentials follow from the free energy as [15]

$$
\begin{gathered}
\Delta \mu=\frac{\delta \mathcal{F}}{\delta \phi}=a \phi+b \phi^{3}-\kappa \nabla^{2} \phi+c\left(\nabla^{2} \phi\right)^{2}+2 \beta_{1} \rho \phi+2 \beta_{2} \phi\left(\nabla^{2} \rho\right)+\beta_{3} \rho\left(\nabla^{2} \phi\right)+\beta_{3} \nabla^{2}(\rho \phi), \\
\Lambda=\frac{\delta \mathcal{F}}{\delta \rho}=\alpha \rho-\lambda \nabla^{2} \rho+\gamma\left(\nabla^{2} \rho\right)^{2}+\beta_{1} \phi^{2}+\beta_{2} \nabla^{2} \phi^{2}+\beta_{3} \phi\left(\nabla^{2} \phi\right) .
\end{gathered}
$$

The derivation of the pressure tensor is slightly more complicated. Considering a linear combination of all symmetric tensors having two or four gradient operators, we find that a suitable choice, which allows the pressure tensor to obey the equilibrium condition $\partial_{\alpha} P_{\alpha \beta}=0$ is

$$
\begin{aligned}
P_{\alpha \beta}= & \left\{p_{L}+c\left[\left(\nabla^{2} \phi\right)^{2}+\partial_{\sigma} \phi \partial_{\sigma} \nabla^{2} \phi\right]+\gamma\left[\left(\nabla^{2} \rho\right)^{2}+\gamma \partial_{\sigma} \rho \partial_{\sigma} \nabla^{2} \rho\right]+\beta_{2}\left[\partial_{\sigma} \phi^{2} \partial_{\sigma} \rho+\phi^{2} \nabla^{2} \rho\right]\right. \\
& \left.+\beta_{3}\left[\partial_{\sigma} \rho \phi \partial_{\sigma} \phi+\rho \phi \nabla^{2} \phi\right]\right\} \delta_{\alpha \beta}+\kappa \partial_{\alpha} \phi \partial_{\beta} \phi-c\left[\partial_{\alpha} \phi \partial_{\beta} \nabla^{2} \phi+\partial_{\beta} \phi \partial_{\alpha} \nabla^{2} \phi\right]+\lambda \partial_{\alpha} \rho \partial_{\beta} \rho \\
& -\gamma\left[\partial_{\alpha} \rho \partial_{\beta} \nabla^{2} \rho+\partial_{\beta} \rho \partial_{\alpha} \nabla^{2} \rho\right]-\beta_{2}\left[\partial_{\alpha} \phi^{2} \partial_{\beta} \rho+\partial_{\alpha} \rho \partial_{\beta} \phi^{2}\right]-\beta_{3}\left[\partial_{\alpha} \rho \phi \partial_{\beta} \phi+\partial_{\alpha} \phi \partial_{\beta} \rho \phi\right](4)
\end{aligned}
$$

where

$$
\begin{aligned}
p_{L}= & \frac{a}{2} \phi^{2}+\frac{3}{4} b \phi^{4}-\kappa \phi\left(\nabla^{2} \phi\right)-\frac{\kappa}{2}(\nabla \phi)^{2}+c \phi\left(\nabla^{2}\right)^{2} \phi-\frac{c}{2}\left(\nabla^{2} \phi\right)^{2}+\frac{\alpha}{2} \rho^{2}-\lambda \rho\left(\nabla^{2} \rho\right)-\frac{\lambda}{2}(\nabla \rho)^{2} \\
& +\gamma \rho\left(\nabla^{2}\right)^{2} \rho-\frac{\gamma}{2}\left(\nabla^{2} \rho\right)^{2}+2 \beta_{1} \rho \phi^{2}+\beta_{2} \phi^{2}\left(\nabla^{2} \rho\right)+\beta_{2} \rho\left(\nabla^{2} \phi^{2}\right)+\beta_{3} \rho \phi\left(\nabla^{2} \phi\right)+\beta_{3} \phi \nabla^{2}(\rho \phi)(5)
\end{aligned}
$$

The lattice Boltzmann scheme is defined in terms of three distribution functions $f_{i}(\mathbf{r}), g_{i}(\mathbf{r})$ and $h_{i}(\mathbf{r})$, each of which evolves during a time step $\Delta t$ according to a single relaxation time Boltzmann equation [16, 17]

$$
f_{i}\left(\mathbf{r}+\mathbf{e}_{i} \Delta t, t+\Delta t\right)-f_{i}(\mathbf{r}, t)=-\frac{1}{\tau}\left[f_{i}(\mathbf{r}, t)-f_{i}^{0}(\mathbf{r}, t)\right],
$$




$$
\begin{aligned}
g_{i}\left(\mathbf{r}+\mathbf{e}_{i} \Delta t, t+\Delta t\right)-g_{i}(\mathbf{r}, t) & =-\frac{1}{\tau_{\phi}}\left[g_{i}(\mathbf{r}, t)-g_{i}^{0}(\mathbf{r}, t)\right], \\
h_{i}\left(\mathbf{r}+\mathbf{e}_{i} \Delta t, t+\Delta t\right)-h_{i}(\mathbf{r}, t) & =-\frac{1}{\tau_{\rho}}\left[h_{i}(\mathbf{r}, t)-h_{i}^{0}(\mathbf{r}, t)\right]
\end{aligned}
$$

where $\tau, \tau_{\phi}$ and $\tau_{\rho}$ are independent relaxation parameters and $\mathbf{e}_{\mathrm{i}}$ are the unit lattice vectors. The distribution functions are related to the physical variables by

$$
n=\sum_{i} f_{i}, \quad n \mathbf{u}=\sum_{i} f_{i} \mathbf{e}_{i}, \quad \phi=\sum_{i} g_{i}, \quad \rho=\sum_{i} h_{i}
$$

where $\mathrm{n}$ is the total density and $\mathbf{u}$ is the mean fluid velocity. These quantities are locally conserved and, therefore, we require that the equilibrium distribution functions $f_{i}^{0}, g_{i}^{0}, h_{i}^{0}$ also fulfil Eqs. (9). The higher moments of $f_{i}^{0}, g_{i}^{0}$ and $h_{i}^{0}$ are defined by imposing the additional requirements

$$
\begin{gathered}
\sum_{i} f_{i}^{0} e_{i \alpha} e_{i \beta}=P_{\alpha \beta}+n u_{\alpha} u_{\beta}, \sum_{i} g_{i}^{0} e_{i \alpha}=\phi u_{\alpha}, \sum_{i} g_{i}^{0} e_{i \alpha} e_{i \beta}=\Gamma_{\phi} \Delta \mu \delta_{\alpha \beta}+\phi u_{\alpha} u_{\beta}, \\
\sum_{i} h_{i}^{0} e_{i \alpha}=\rho u_{\alpha}, \sum_{i} h_{i}^{0} e_{i \alpha} e_{i \beta}=\Gamma_{\rho} \Lambda \delta_{\alpha \beta}+\rho u_{\alpha} u_{\beta}
\end{gathered}
$$

where $\Gamma_{\phi}$ and $\Gamma_{\rho}$ are mobilities. The equilibrium distribution functions are defined as usual in terms of an expansion in the velocity $\mathbf{u}[9,10]$. We use a 9 -velocity model on a square lattice to obtain the results presented here.

These definitions lead to the continuum equations which follow from expanding Eqs. (6), (7) and (8) to $\mathrm{O}\left(\Delta t^{2}\right) 10$

$$
\begin{gathered}
\partial_{t} n+\partial_{\alpha}\left(n u_{\alpha}\right)=0, \partial_{t}\left(n u_{\alpha}\right)+\partial_{\beta}\left(n u_{\alpha} u_{\beta}\right)=-\partial_{\beta} P_{\alpha \beta}+\nu \nabla^{2}\left(n u_{\alpha}\right)+\partial_{\alpha}\left[\lambda(n) \partial_{\gamma}\left(n u_{\gamma}\right)\right], \\
\partial_{t} \phi+\partial_{\alpha}\left(\phi u_{\alpha}\right)=\Gamma_{\phi} \Theta_{\phi} \nabla^{2} \Delta \mu-\Theta_{\phi} \partial_{\alpha}\left(\frac{\phi}{n} \partial_{\beta} P_{\alpha \beta}\right) \\
\partial_{t} \rho+\partial_{\alpha}\left(\rho u_{\alpha}\right)=\Gamma_{\rho} \Theta_{\rho} \nabla^{2} \Lambda-\Theta_{\rho} \partial_{\alpha}\left(\frac{\rho}{n} \partial_{\beta} P_{\alpha \beta}\right),
\end{gathered}
$$

where

$$
\nu=\frac{(2 \tau-1)}{6}(\Delta t), \lambda(n)=\left(\tau-\frac{1}{2}\right) \Delta t\left(\frac{1}{2}-\frac{d p_{0}}{d n}\right), \Theta_{\phi}=\Delta t\left(\tau_{\phi}-\frac{1}{2}\right), \Theta_{\rho}=\Delta t\left(\tau_{\rho}-\frac{1}{2}\right) .
$$

Dynamical Behaviour. - Unless otherwise stated the simulations were run with $a=-1$, $b=c=1, \kappa=0.1, \alpha=\lambda=\gamma=1, \beta_{1}=0, \beta_{2}=-0.2, \beta_{3}=0.4, \tau=100, \tau_{\phi}=\tau_{\rho}=$ $(1+1 / \sqrt{3}) / 2[9]$ and with units in which $\Delta t=1$.

Above the critical temperature $(a>0)$ the equilibrium configuration is a mixture of the three components, with constant $n, \phi=0$ and $\rho=0$. For small variations in $\phi$, Eq. (13) can be linearised about $\phi=0$ resulting in a convection-diffusion equation with diffusion constant $D_{\phi}=a \Gamma_{\phi} \Theta_{\phi}$. Similarly for small variations in $\rho$, Eq. (14) can be linearised resulting in a diffusion equation with $D_{\rho}=\alpha \Gamma_{\rho} \Theta_{\rho}$. Following [9], we tested these predictions by measuring $D_{\phi}$ and $D_{\rho}$ as functions of $\Gamma_{\phi} \Theta_{\phi}$ and $\Gamma_{\rho} \Theta_{\rho}$ respectively by monitoring the decay of a sinusoidal perturbation. The measured values are shown in Fig. 1 for different values of $a$ and $\alpha$. Agreement with the predicted values is very good. 

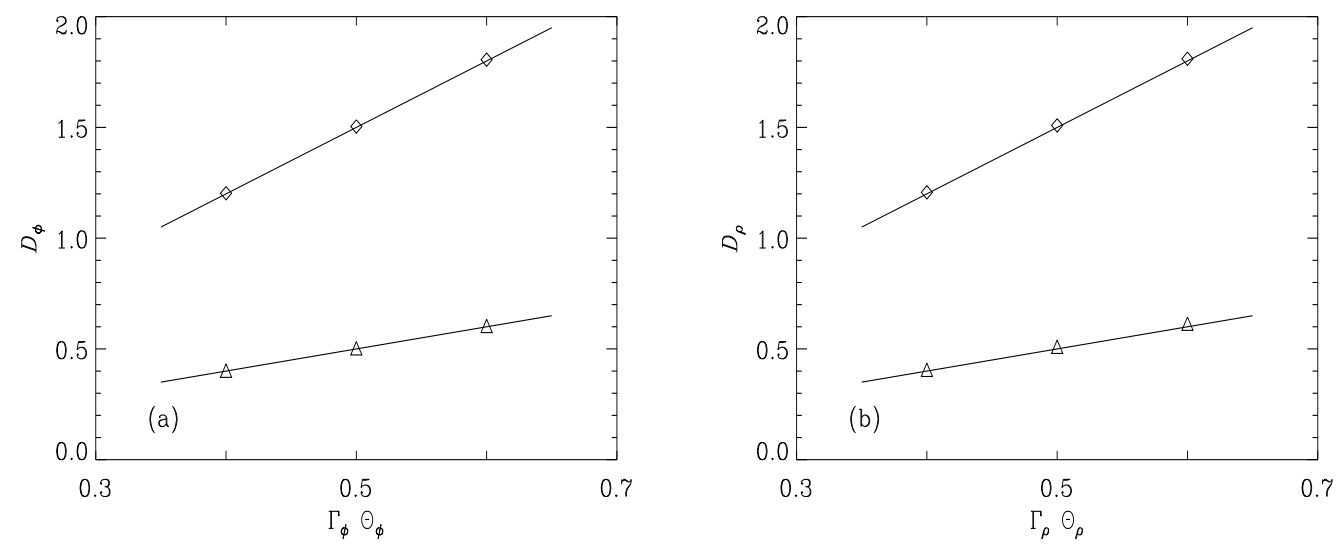

Fig. 1. - Diffusion constant $D$ as a function of mobility $\Gamma \Theta$ for temperatures above critical for (a) the oil-water density difference and (b) the surfactant density. The full lines are analytic results which follow from linearising Eqs. (13) and (14). Data points were obtained by following the decay of a small sinusoidal perturbation.
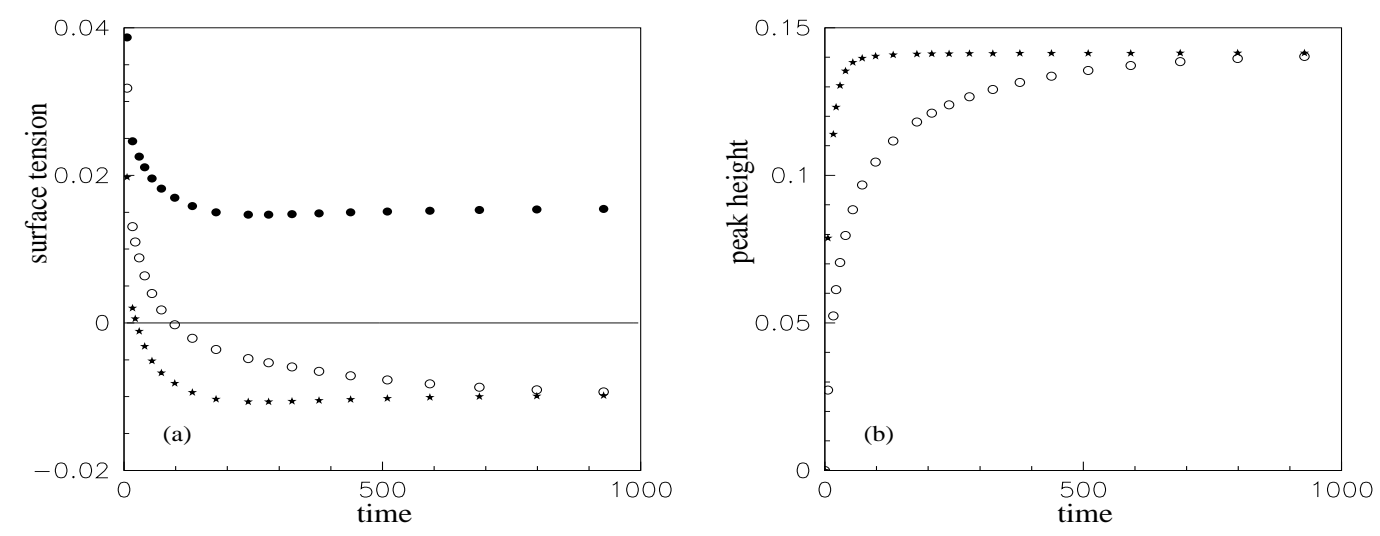

Fig. 2. - Variation of (a) the surface tension of a flat interface, (b) the maximum surfactant density at the interface with time for different values of the surfactant mobility: $\bullet \Gamma_{\rho} \Theta_{\rho}=0$, ० $\Gamma_{\rho} \Theta_{\rho}=0.1$, $\star \Gamma_{\rho} \Theta_{\rho}=2.5$. The parameter $\kappa$ is fixed to the value -1.15 and $\Gamma_{\phi} \Theta_{\phi}=0.1$.

Below the critical temperature $(a<0)$ the binary mixture phase separates into two distinct phases, symmetric about $\phi=0$. Oil-water interfaces are now formed in the system. We next consider the diffusion of the surfactant towards these interfaces. A system set-up to contain an oil-water interface that was initially a hyperbolic tangent was initialised with $\rho=0$ and and then allowed to evolve. Figure 2a shows the surface tension plotted as a function of time for three different values of the surfactant mobility. For zero mobility the interface relaxes to its equilibrium shape thus causing a decrease in the surface tension. However, no surfactant 

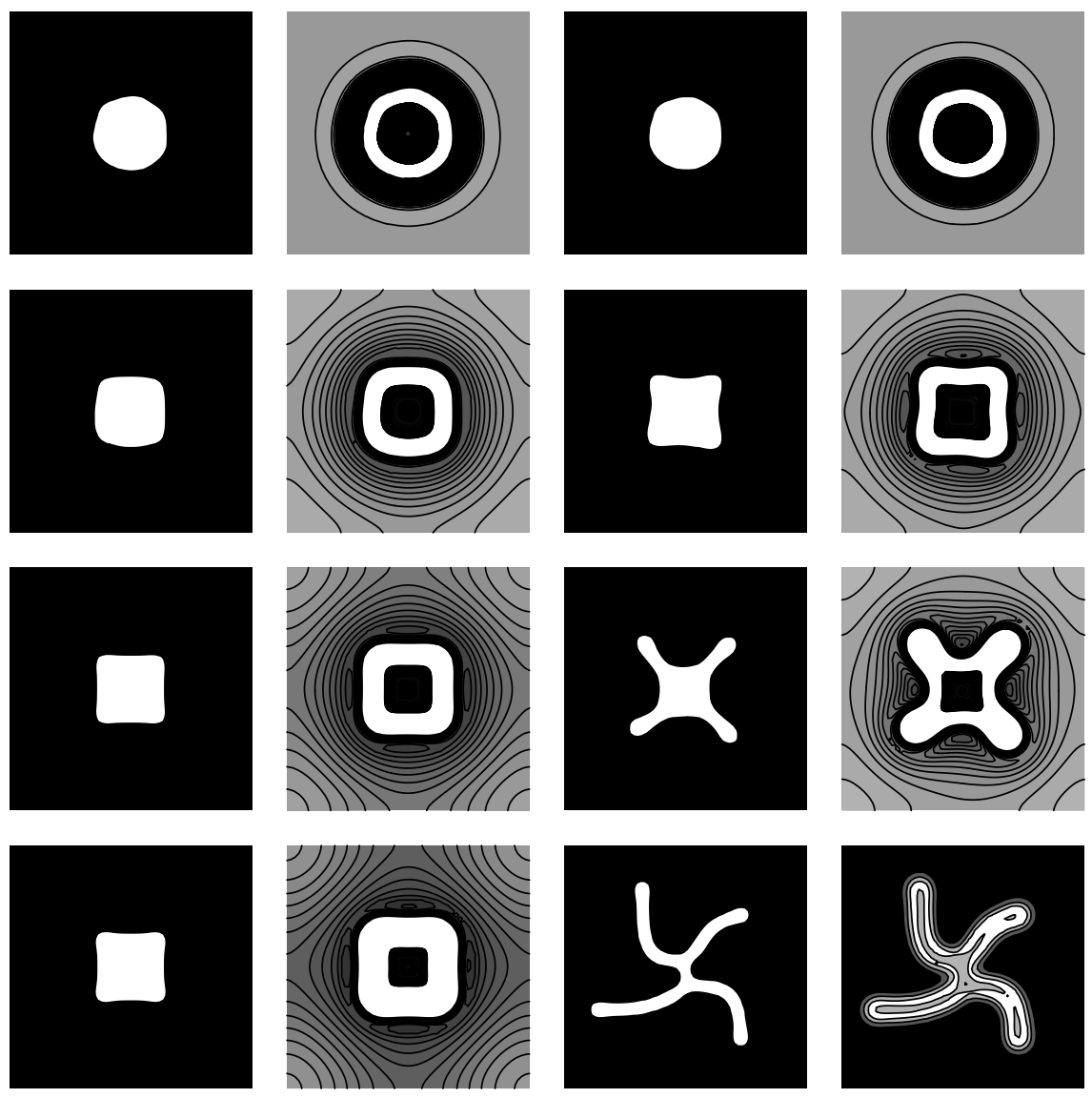

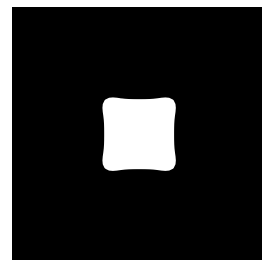

(a)

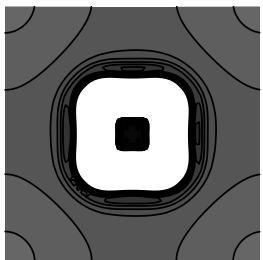

(b)

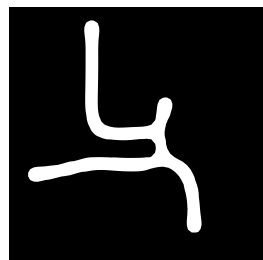

(c)

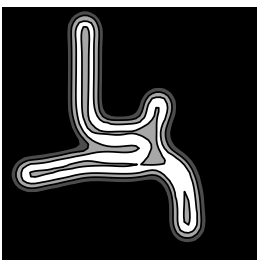

(d)

Fig. 3. - Spontaneous emulsification of a droplet as surfactant diffuses to the interface. Results are given for the evolution with time (from top, $\mathrm{t}=46,591,1252,2652,5615$ ) of (a) the oil-water density difference $\phi$ for high viscosity; (b) the surfactant density $\rho$ (grey-scaling from black $\Rightarrow$ white corresponds to minimum $\rho \Rightarrow$ maximum $\rho$ ) for high viscosity; (c) $\phi$ for low viscosity; (d) $\rho$ for low viscosity. $\kappa=-1.15$ and $\Gamma_{\phi} \Theta_{\phi}=\Gamma_{\rho} \Theta_{\rho}=0.1$.

diffuses to the interface and the surface tension remains positive. For finite mobility however the surfactant can diffuse to the interface and the final surface tension is negative. The rate at which the final value is achieved clearly depends on the value of the diffusion constant. Similar considerations apply to the increase in the peak value of the surfactant density $\rho$ at the interface as a function of time (Fig. 2b). 
Once the surface tension has become negative the interface should become unstable 13 . Figure 3 shows the evolution with time of a drop of oil in water which is initially approximately circular. (Small randomness in the radius is necessary or the drop remains metastable.) The evolution of both the $\phi$ and $\rho$ fields are shown. Small perturbations on the surface of the drop grow to form lamellae of a width appropriate to that minimising the free energy. The budding of the drop initially reflects the symmetry of the underlying lattice. We believe this to be a consequence of the sharpness of the interface relative to the lattice spacing.

Figure 3 compares the spontaneous emulsification at high $(\tau=100)$ and low $(\tau=0.585)$ viscosities. The change in viscosity makes a striking difference to the speed at which the lamellae form.

Summary. - We have described a lattice Boltzmann model for ternary mixtures, such as oil-water-surfactant systems. The model includes hydrodynamic and diffusive modes and controls the fluid equilibrium via a chosen input free energy. The viscosity and diffusivities of the fluid phases can be changed thus allowing a study of their effects on the self-assembly and rheology of the amphiphilic fluid. In particular we presented results for the effect of surface diffusion on the rate of change of the interfacial tension and for the spontaneous emulsification which results as the tension becomes negative.

The model is not trivial and much work remains to be done to explore its physical and numerical properties within a wide dynamic and static parameter space. A well-defined equilibrium and the ability to impose rather than measure transport coefficients are particularly useful in this task. Extensions to three dimensions and a closer comparison to experimental results are important endeavours.

We thank Enzo Orlandini and Peter Coveney for helpful discussions.

\section{REFERENCES}

[1] Gelbart W. M., Ben-Shaul A. and Roux D., editors, Micelles, Membranes, Microemulsions and Monolayers, Springer-Verlag, Berlin 1994.

[2] Gompper G. and Schick M., in Phase Transition and Critical Phenomena, edited by C. Domb and J. L. Lebowitz, Vol. 16 (Academic Press, London) 1994 pp. 1-176.

[3] Hennes M. and Gompper G., Phys. Rev. E, 54 (1996) 3811.

[4] Pätzold G. and Dawson K. A., Phys. Rev. E, 52 (1995) 6908.

[5] Laradji M., Mouritsen O. G., Toxvaerd S. and Zuckerman M.J., Phys. Rev. E, 50 (1994) 1243.

[6] Emerton A. N., Coveney P. V. and Boghosian B. M., Phys. Rev. E, 55 (1997) 708.

[7] Weig F. W. J., Coveney P. V. and Boghosian B. M, Phys. Rev. E, 56 (1997) 6877.

[8] Chen S. and Doolen G. D., Ann. Rev. Fluid. Mech., 30 (1998) 329

[9] Orlandini E., Swift M. R. and Yeomans J. M., Europhys. Lett., 32 (1995) 463.

[10] Swift M. R., Orlandini E., Osborn W. R. and Yeomans J. M., Phys. Rev. E, 54 (1996) 5041 .

[11] Gonnella G., Orlandini E. and Yeomans J. M., Phys. Rev. Lett., 78 (1997) 1695.

[12] Theissen O., Gompper G. and Kroll D.M., Europhys. Lett., 42 (1998) 419.

[13] Granek R., Ball R. C. and Cates M. E., J. Phys. II France, 3 (1993) 829.

[14] Gompper G and Schick M., Phys. Rev. E, 49 (1994) 1478.

[15] Rowlinson J. S. and Widom B., Molecular Theory of Capillarity, Oxford 1982.

[16] Bhatnagar P. L., Gross E. P. and Krook M., Phys. Rev., 94 (1954) 511.

[17] Chen H., Chen S. and Matthaeus W. H., Phys. Rev. A, 45 (1992) R5339. 